\title{
MORTALIDADE POR QUEDA EM IDOSOS: UMA REVISÃO INTEGRATIVA
}

Fall mortality in the elderly: an integrative review

Muerte por caída en los ancianos: una revisión integradora

Denise Guerra Wingerter $\bullet$ Analista de Sistemas • Epidemiologista • Especialista em Análise de Situação em Saúde • Mestre em Demografia e doutoranda em Saúde Coletiva da Universidade Federal do Rio Grande do Norte-UFRN • E-mail: denisegw@gmail.com

Isabelle Ribeiro Barbosa • Professora Doutora do Programa de Pós Graduação em Saúde Coletiva da UFRN・E-mail: isabelleribeiro@oi.com.br

Luana Kelle Batista Moura • Professora do Centro Universitário UNINOVAFAPI. Teresina PI • E-mail: luana_moura19@hotmail.com

Rafael Fábio Maciel • UNIFESP • Professor Doutor da pós-graduação em Informática em Saúde. E-mail: rfmaciel@uol.com.br

Maria do Socorro Costa Feitosa Alves • Professora titular da UFRN • E-mail: socorrocfa@hotmail.com

Autora responsável pela correspondência:

Denise Guerra Wingerter • E-mail: denisegw@gmail.com 


\section{RESUMO}

Introdução: A Organização Mundial de Saúde avalia que aproximadamente $28 \%$ a $42 \%$ das pessoas acima de 65 anos sofrem quedas anualmente, e 5 a $10 \%$ destas evoluem para óbito. Objetivo: Verificar o perfil epidemiológico de óbitos por quedas em idosos entre 2007 e 2016. Método: Busca de artigos com descritores: Idoso; Óbito; Envelhecimento; Perfil de Saúde; Acidentes por Quedas; nas bases de dados CAPES, SciELO, BVS; entre 2007 e 2016; utilizando Idiomas: Inglês, português, espanhol; Incluídos artigos cujo objetivo avaliou os óbitos por quedas em idosos excluindo os que não possuíam informações sobre quedas ou continham a informação queda como fator de risco para mortalidade sem o desfecho óbito. Resultados: Triados oito artigos, seis utilizaram estudos retrospectivos entre 1996 a 2011 tendo o Sistema de Informação de Mortalidade (SIM) como base. Foram encontrados percentuais gerais de óbitos por quedas em idosos entre 0,61\% e 0,69\% e 7,15\% das causas externas. A Mortalidade Específica por quedas variou entre 15 a 29\%. O Coeficiente de Mortalidade Específico variou de 24,06 a 35,83/100000hab. Gênero, raça, estado civil e escolaridade não apresentaram avaliações que permitissem análise mais efetiva. Todos os estudos apontaram que quanto maior a idade, maior o risco de óbito por queda. Os estudos baseados no SIM apontaram para a limitação da qualidade do dado coletado, onde o óbito por queda pode estar mascarado por outras causas. Conclusões: Encontradas informações insuficientes para traçar o perfil epidemiológico da população idosa que vem a óbito por quedas, assim como disparidades entre taxas gerais e específicas de mortalidade. Mostra-se importante a qualificação da causa básica do óbito no preenchimento das informações do SIM, uma vez que o preenchimento incorreto pode mascarar a magnitude deste agravo, suas proporções e coeficientes, prejudicando a avaliação e alocação de políticas públicas que possam reduzir esta mortalidade.

Palavras-Chave: Saúde do Idoso, Mortalidade, Acidentes por quedas. 


\section{ABSTRACT}

Introduction: The World Health Organization estimates that approximately $28 \%$ to $42 \%$ of people over 65 suffer falls annually, and 5 to $10 \%$ of them die. Objective: To verify the epidemiological profile of deaths due to falls in the elderly between 2007 and 2016. Method: Search for articles with descriptors: Elderly; Death; Aging; Health Profile; Falls Accidents; in the CAPES, SciELO, BVS databases; between 2007 and 2016; using Languages: English, Portuguese, Spanish; Included were articles whose objective evaluated deaths from falls in the elderly, excluding those who did not have information about falls or contained information about falls as a risk factor for mortality without the outcome of death. Results: Screened eight articles, six used retrospective studies between 1996 to 2011 using the Mortality Information System (SIM) as a database. General percentages of deaths from falls were found in the elderly between $0.61 \%$ and $0.69 \%$ and $7.15 \%$ of external causes. Specific mortality from falls ranged from 15 to 29\%. The Specific Mortality Coefficient ranged from 24.06 to 35.83 / 100000hab. Gender, race, marital status and education did not present evaluations that would allow more effective analysis. All studies pointed out that the older the age, the greater the risk of death from falls. Studies based on SIM pointed to the limitation of the quality of the data collected, where death from falls may be masked by other causes. Conclusions: Insufficient information was found to trace the epidemiological profile of the elderly population that dies from falls, as well as disparities between general and specific mortality rates. It is important to qualify the basic cause of death when filling out SIM information, since incorrect filling can mask the magnitude of this condition, its proportions and coefficients, impairing the evaluation and allocation of public policies that can reduce this mortality.

Keywords: Elderly Health, Mortality, Accidents due to falls. 


\section{RESUMEN}

Introducción: la Organización Mundial de la Salud estima que aproximadamente $28 \%$ al $42 \%$ de las personas mayores de 65 años sufren caídas anuales, y del 5 al $10 \%$ de ellas mueren. Objetivo: verificar el perfil epidemiológico de las muertes por caídas en los ancianos entre 2007-2016. Método: buscar artículos con descriptores: ancianos; Muerte; Envejecimiento Perfil de salud; Accidentes de caídas; en las bases de datos CAPES, SciELO, BVS; entre 2007 y 2016; idiomas: inglés, portugués, español; Se incluyeron artículos cuyo objetivo evaluó las muertes por caídas en los ancianos, excluyendo a aquellos que no tenían información sobre caídas o que contenían información sobre caídas como factor de riesgo de mortalidad sin el resultado de la muerte. Resultados: Ocho artículos encontrados, seis utilizaron estudios retrospectivos entre 1996 y 2011 utilizando el Sistema de Información de Mortalidad (SIM). Porcentajes generales de muertes entre $0,61 \%$ y $0,69 \%$ y $7,15 \%$ de causas externas. La mortalidad específica por caídas osciló entre 15 y 29\%. El coeficiente de mortalidad específico varió de 24.06 a 35.83 / 100000hab. El género, la raza, el estado civil y la educación no presentaron evaluaciones que permitieran un análisis más efectivo. Todos los estudios señalaron que cuanto mayor es la edad, mayor es el riesgo de muerte por caídas. Los estudios basados en SIM señalaron la limitación de la calidad de los datos, donde la muerte por caídas puede estar enmascarada por otras causas. Conclusiones: se encontró información insuficiente para rastrear el perfil epidemiológico de la población anciana que muere por caídas, e disparidades entre las tasas de mortalidad general y específica. Es importante calificar la causa básica de muerte en la SIM, ya que un llenado incorrecto puede enmascarar la magnitud de esta condición, perjudicando la evaluación y asignación de políticas públicas que puden reducir esta mortalidad.

Palabras clave: Ancianos, muerte, Accidentes por caídas. 


\section{Introdução}

Envelhecer é um processo natural, dinâmico e progressivo, onde acontecem diversas alterações físicas, bioquímicas, funcionais e morfológicas, ocorrendo com o tempo a redução da capacidade de adaptação funcional às situações do cotidiano, tornando nosso organismo mais suscetível às enfermidades e às casualidades do dia-a-dia ${ }^{1}$.

No Brasil, considera-se pessoa idosa aquela que tem 60 anos ou mais, independentemente de seu estado biológico, psicológico e social ${ }^{2}$, porém o conceito de idade é multidimensional e não é uma boa medida do desenvolvimento humano, uma vez que a idade e o processo de envelhecimento possui outras dimensões e significados que extrapolam as dimensões da idade cronológica, variando com estilos de vida e decisões ao longo da vida e desta forma, duas pessoas não envelhecem de maneira idêntica ${ }^{3}$.

Nas últimas décadas, o mundo vem observando um processo de envelhecimento de suas populações e aumento da expectativa de vida, com quedas bruscas das taxas de natalidade e de mortalidade, o que acarretará a médio e longo prazo, populações mais envelhecidas. No Brasil, a expectativa do Instituto Brasileiro de Geografia e Estatística (IBGE) é de que a população com mais de 60 anos de idade seja aproximadamente 11\% da população geral até o ano de $2020^{4}$.

Esse processo de envelhecimento populacional demandará cada vez mais, produtos e serviços específicos para esta parcela crescente da população além de ajustes na estrutura social, econômica, política e cultural da sociedade, uma vez que os idosos possuem demandas específicas para obtenção de adequadas condições de vida ${ }^{2}$.

Uma das principais preocupações relacionadas ao envelhecimento são as morbidades que acometem estes indivíduos, e a instabilidade postural e as 
quedas são consideradas síndromes geriátricas que englobam as alterações de saúde mais comuns nos idosos, constituindo um dos principais problemas clínicos e de saúde pública devido à sua alta incidência, às consequentes complicações para a saúde e aos altos custos assistenciais ${ }^{5}$.

A Organização Mundial de Saúde define a queda como "vir a inadvertidamente ficar no solo ou em outro nível inferior, excluindo mudanças de posição intencionais para se apoiar em móveis, paredes ou outros objetos", e avalia que em torno de $28 \%$ a $35 \%$ das pessoas com mais de 65 anos de idade sofrem quedas a cada ano, proporção que é acentuada para 32\% a 42\% para as pessoas com mais de 70 anos ${ }^{6}$.

Embora indivíduos de todas as idades apresentem riscos de sofrer quedas, para os idosos as quedas possuem um significado mais relevante, uma vez que "podem levá-lo à incapacidade e morte", possuindo assim um alto custo psicológico, social e econômico, e impacta diretamente na diminuição da autonomia do idoso e da sua independência?

Estima-se que um em cada três indivíduos com mais de 65 anos venha a cair e que entre os idosos que caem, um em cada vinte sofram uma fratura ou necessitem de internação ${ }^{7}$. Dentre os mais idosos (80 anos e mais) aproximadamente $40 \%$ caem a cada ano, sendo as quedas mais frequentes nos idosos que moram em asilos e casas de repouso, chegando a $50 \%{ }^{6}$.

Segundo levantamento de Terra et al (2013), as quedas em idosos estão associadas a altas taxas de mortalidade, uma vez que de 5 a $10 \%$ das quedas evoluem para óbito, e este percentual aumenta para $30 \%$ em quedas de idosos institucionalizados ${ }^{7}$.

O conhecimento das suas consequências físicas e sociais das quedas em dosos, assim como as taxas de óbito, é de extrema importância, uma vez que ossibilita auxiliar no planejamento e delineamento de estratégias preventivas e de reabilitação de tais repercussões. 
Assim, o objetivo deste estudo foi realizar uma revisão bibliográfica da literatura através da seleção e análise criteriosa de artigos científicos que investigaram os óbitos por quedas em idosos visando verificar o perfil epidemiológico e fatores de risco destes eventos.

\section{Metodologia}

Processo de revisão integrativa da literatura referente à mortalidade em idosos tendo a queda como causa básica de óbito, através de estudos originais disponibilizados em periódicos acadêmicos. Neste contexto, os artigos foram lidos e selecionados criteriosamente de acordo com o seguinte método de trabalho:

1. Identificação de Descritores: Pesquisa por palavras-chave no site no site DeCS - Descritores em Ciências da Saúde (http://decs.bvs.br) com a finalidade de identificar os descritores do tema estudado, sendo selecionadas os seguintes descritores:

- Idoso (Aged, Elderly-Inglês, Anciano-Espanhol);

- Óbito (death - inglês, muerte - espanhol);

- Envelhecimento (aging - inglês, envejecimiento - espanhol);

- Perfil de Saúde (Health Profile - inglês, Perfil de salud - espanhol);

- Acidentes por Quedas (Accidental Falls-inglês, Accidentes por Caídas espanhol)

- Saúde do Idoso (Health of the Elderly - Inglês, Salud del Anciano Espanhol)

Seleção de Bases de dados: As buscas foram realizadas nas seguintes bases de dados bibliográficas: Portal de periódicos CAPES, Portal SciELO - Scientific Electronic Library Online, e Portal BVS Salud. Estes portais englobam a maior 
parte de periódicos e informações bibliográficas disponível na web, e ao final da pesquisa, foram identificadas e excluídas as referências duplicadas

3. Temporalidade: Foram selecionados artigos publicados nos últimos dez anos, (entre os anos de 2007 e 2016), a fim de se recolher material bibliográfico recente e mais antigo.

4. Idiomas: Foram selecionados artigos escritos em inglês, português ou espanhol. Outras línguas foram desprezadas pela impossibilidade de uma leitura crítica adequada.

5. Termos livres: Além dos descritores, foram também utilizados termos livres, uma vez que foram encontrados problemas e diferenças nos processos de indexação nas bases de dados bibliográficas. Assim, optou-se por buscas com descritores (termos controlados) e buscas com termos livres, a fim de verificar qual a melhor estratégia para recuperar as informações desejadas.

6. Critérios de inclusão e exclusão: Foram incluídos todos os artigos de revisão e artigos originais indexados no período entre primeiro de janeiro de 2007 e 30 de agosto de 2016, independente do delineamento e do número de indivíduos estudados, que continham em seu objetivo avaliar os óbitos por quedas em idosos. Foram excluídos os estudos que não possuíam informações sobre quedas ou continham somente a informação da queda como fator de risco para mortalidade em idosos, sem o desfecho óbito.

7. String de pesquisa: As linhas de pesquisa utilizadas foram em português e inglês, com as palavras-chave: [(Accidental falls) AND (Aged) AND (Death)] e [(Acidentes por Quedas ) AND (idoso) AND (óbito)].

8. A busca de artigos e a seleção de artigos final foi realizada por um autor. 


\section{ciência
pural}

\section{Resultados e discussão}

A busca inicial utilizando as strings nos motores de busca supracitados com seus respectivos filtros, resultou em dezoito artigos na BVS:Salud, cinco no Periódico capes, três artigos no motor Scielo.

Foram excluídos oito artigos duplicados e dez artigos que citavam a queda no idoso sem necessariamente o desfecho óbito, conforme figura 1.

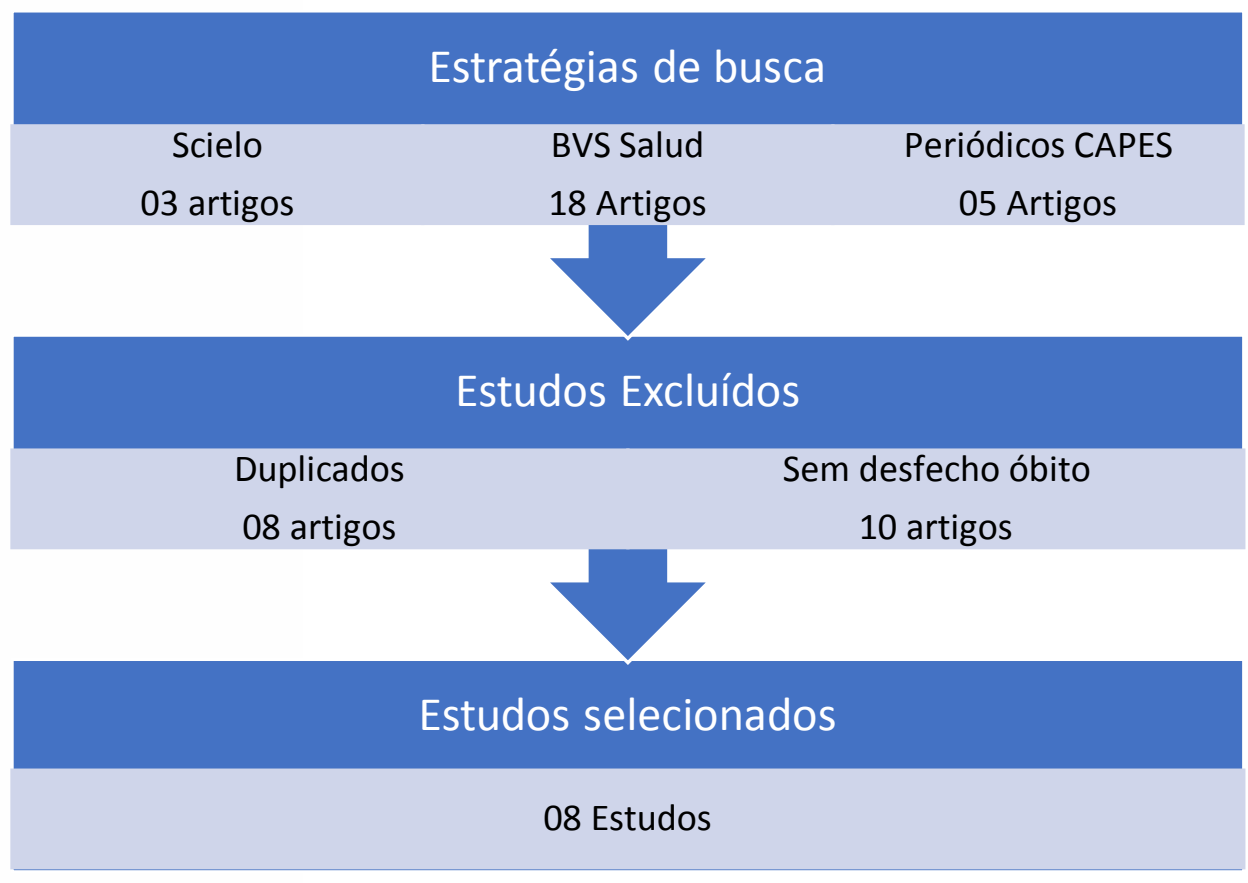

Figura 1 - Fluxograma do processo de seleção dos artigos pesquisados

Após a triagem por artigos que tratassem ou abordassem especificamente o óbito em idosos por quedas, foram selecionados oito artigos em que haviam referência direta sobre óbitos por queda em idosos no objetivo, assunto, resumo ou título, conforme listados na Tabela 1. 
Tabela 1 - Artigos selecionados nas bases de dados BVS Salud, Periódicos Capes

(2007-2016), sobre ocorrência de óbito por quedas em idosos, Brasil.

\begin{tabular}{|c|c|c|c|}
\hline $\begin{array}{l}\text { Título do } \\
\text { Artigo/ } \\
\text { Referência }\end{array}$ & Objetivos & Resultados & $\begin{array}{l}\text { Desenho do estudo e } \\
\text { período de coleta }\end{array}$ \\
\hline $\begin{array}{l}1 \text { Rosa et al } \\
(2015)^{8}\end{array}$ & $\begin{array}{l}\text { Traçar o perfil dos idosos que } \\
\text { foram a óbito por queda no } \\
\text { Rio Grande do Sul no período } \\
\text { de } 2006 \text { a 2011, utilizando o } \\
\text { Sistema de Informaçoses sobre } \\
\text { Mortalidade. Constatou-se } \\
\text { que o coeficiente de } \\
\text { mortalidade por queda } \\
\text { aumentou de } 2006 \text { para } 2011 \\
\text { naquele estado, sendo mais } \\
\text { elevado para os idosos de } 80 \\
\text { anos ou mais, resultados de } \\
\text { relevância para o } \\
\text { desenvolvimento de políticas } \\
\text { públicas para o idoso. }\end{array}$ & $\begin{array}{l}\text { A chance de óbitos por } \\
\text { queda nos idosos é } \\
\text { significativamente maior } \\
\text { para o gênero feminino, } \\
\text { acima de } 69 \text { anos, brancos, } \\
\text { viúvos ou solteiros. } \\
\text { Houve aumento de } 41,8 \% \\
\text { nos coeficientes de } \\
\text { mortalidade específicos } \\
\text { por queda no período. } \\
\text { Maior coeficiente em } 2011 \\
31,56 / 100.000 \text {. }\end{array}$ & $\begin{array}{l}\text { Estudo descritivo de } \\
\text { série temporal com } \\
\text { dados do Sistema de } \\
\text { Informações sobre } \\
\text { Mortalidade entre } \\
2006 \text { a 2011. }\end{array}$ \\
\hline $\begin{array}{l}2 \text { Maciel et } \\
\text { al }(2010)^{9}\end{array}$ & $\begin{array}{l}\text { Descrição e caracterização da } \\
\text { mortalidade por quedas em } \\
\text { idosos [...] em residentes das } \\
\text { capitais brasileiras, no } \\
\text { período de } 1996 \text { a } 2005 . \\
\text { Estudo retrospectivo com } \\
\text { dados secundários do } \\
\text { Ministério da Saúde sobre } \\
\text { mortalidade por quedas em } \\
\text { idosos. }\end{array}$ & $\begin{array}{l}\text { As causas externas } \\
\text { levaram a óbito, no Brasil, } \\
41.054(3 \%) \text { idosos; destes, } \\
\text { em média, } 22,5 \% \text { ( }=9.249) \\
\text { foram codificados como } \\
\text { quedas. Verificou-se que } \\
\text { os maiores coeficientes de } \\
\text { mortalidade por queda } \\
\text { foram registrados nos } \\
\text { anos de } 2004 \text { e } 2005 \text { (33,2 e } \\
31,5 / 100.000 \\
\text { respectivamente). } \\
\text { Predominância do sexo } \\
\text { masculino, em Brasília e } \\
\text { nas capitais Vitória, } \\
\text { Curitiba, Aracaju e } \\
\text { Goiânia. }\end{array}$ & $\begin{array}{lr}\text { Estudo retrospectivo } \\
\text { de dados secundários } \\
\text { sobre mortalidade } \\
\text { com dados } & \text { do } \\
\text { Sistema } & \text { de } \\
\text { Informações } & \text { sobre } \\
\text { Mortalidade } & \text { entre } \\
1996 \text { e 2005 } & \end{array}$ \\
\hline
\end{tabular}




\begin{tabular}{|c|c|c|c|}
\hline $\begin{array}{l}\text { Título do } \\
\text { Artigo/ } \\
\text { Referência }\end{array}$ & Objetivos & Resultados & $\begin{array}{l}\text { Desenho do estudo e } \\
\text { período de coleta }\end{array}$ \\
\hline $\begin{array}{ll}3 \text { Evans } & \text { D } \\
\text { et } & \text { al } \\
(2015)^{10} & \end{array}$ & 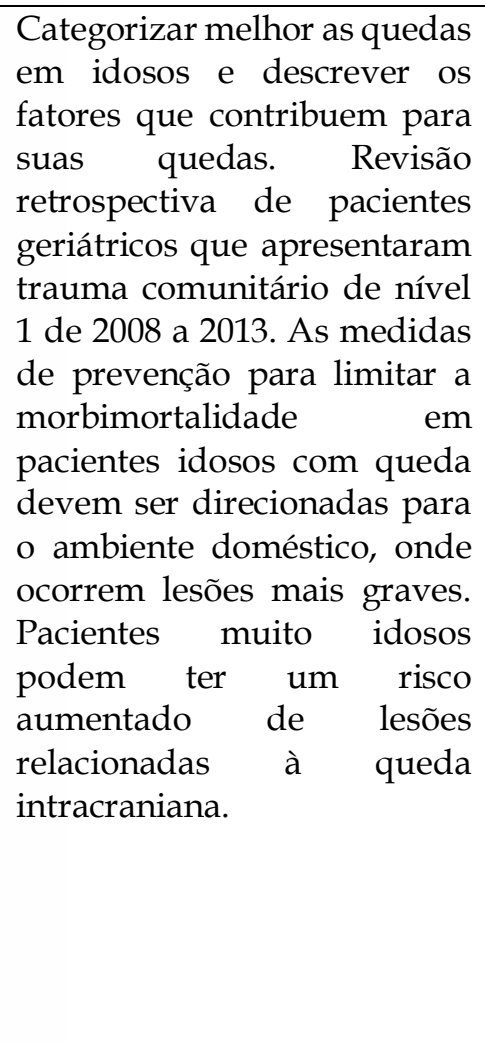 & $\begin{array}{l}\text { Noventa e cinco pacientes } \\
\text { morreram ou foram } \\
\text { internados em decorrência } \\
\text { de suas quedas (14,7\%), } \\
\text { dos quais } 88 \text { vieram de } \\
\text { casa. Controlando o escore } \\
\text { de gravidade das lesões, } \\
\text { morar em casa foi um fator } \\
\text { de risco independente } \\
\text { para mortalidade } \\
\text { relacionada à queda. } \\
\text { Comparando-se os idosos } \\
\text { (65-79 anos; } n=274) \text { e os } \\
\text { muito idosos (idade } \geq 80 \\
\text { anos; }=376), \text { não houve } \\
\text { diferenças no Índice de } \\
\text { Gravidade da Lesão (P = } \\
\text { 0,33), probabilidade de } \\
\text { morte (P = }(\mathrm{P} \text {.49), } \\
\text { probabilidade de lesão na } \\
\text { coluna } \mathrm{C}(\mathrm{P}=1.0) \text { ou } \\
\text { probabilidade de outra } \\
\text { lesão esquelética axial ou } \\
\text { óssea longa }(\mathrm{P}=.23-1.0) \text {. }\end{array}$ & $\begin{array}{l}\text { Estudo retrospectivo } \\
\text { de quedas em idosos } \\
\text { em hospital de } \\
\text { trauma entre } 2008 \text { a } \\
2013\end{array}$ \\
\hline $\begin{array}{l}\text { 4 Coutinho } \\
\text { E.S.Fet al } \\
(2012)^{11}\end{array}$ & $\begin{array}{l}\text { Caracterizar o perfil de } \\
\text { mortalidade de idosos que } \\
\text { sofreram fraturas graves, } \\
\text { hospitalizados devido à } \\
\text { fratura decorrente de queda, } \\
\text { e idosos da população } \\
\text { acompanhados por um ano. } \\
\text { Esses grupos foram pareados } \\
\text { por idade, sexo, momento da } \\
\text { hospitalização do caso e } \\
\text { vizinhança. Obitos } \\
\text { identificados por } \\
\text { relacionamento probabilístico } \\
\text { do banco de dados do estudo } \\
\text { com a base de dados de } \\
\text { mortalidade do estado. }\end{array}$ & $\begin{array}{l}\text { A mortalidade acumulada } \\
\text { em um ano foi de } 25,2 \% \text { e } \\
4 \% \text { para idosos com e sem } \\
\text { fratura grave, } \\
\text { respectivamente. } \\
\text { distribuição dos óbitos } \\
\text { não foi homogênea ao } \\
\text { longo do tempo de } \\
\text { seguimento. Dois terços } \\
\text { das mortes entre paciente } \\
\text { ocorreram no } 1^{\circ} \text { trimestre } \\
\text { após a fratura, enquanto } \\
\text { que entre os controles os } \\
\text { óbitos foram mais tardios. }\end{array}$ & $\begin{array}{lr}\text { Estudo de } & \text { caso- } \\
\text { controle } & \text { de } \\
\text { mortalidade } & \text { por } \\
\text { queda e mortalidade } \\
\text { geral em } & \text { idosos. } \\
\text { Perído } & \text { não } \\
\text { informado. } & \end{array}$ \\
\hline $\begin{array}{l}5 \text { Gomes et } \\
\text { al }(2010)^{12}\end{array}$ & $\begin{array}{l}\text { Caracterizar o perfil dos } \\
\text { óbitos em idosos em Minas } \\
\text { Gerais, Brasil, no período de } \\
1999 \text { a 2008, com dados do } \\
\text { Sistema de Informações sobre } \\
\text { Mortalidade do Ministério da } \\
\text { Saúde. }\end{array}$ & $\begin{array}{l}\text { Coeficientes crescentes de } \\
\text { mortalidade em idosos } \\
\text { durante os anos } \\
\text { estudados. Principais } \\
\text { causas as quedas e os } \\
\text { acidentes de transporte. }\end{array}$ & $\begin{array}{l}\text { Estudo descritivo de } \\
\text { série temporal } \\
\text { baseado em dados do } \\
\text { Sistema de } \\
\text { Informações sobre } \\
\begin{array}{ll}\text { Mortalidade entre } \\
\text { 1999 a 2008. }\end{array} \\
\end{array}$ \\
\hline
\end{tabular}




\begin{tabular}{|c|c|c|c|}
\hline $\begin{array}{l}\text { Título do } \\
\text { Artigo/ } \\
\text { Referência }\end{array}$ & Objetivos & Resultados & $\begin{array}{l}\text { Desenho do estudo e } \\
\text { período de coleta }\end{array}$ \\
\hline $\begin{array}{l}6 \text { Araújo } \\
\text { A.M.M., et } \\
\text { al }(2014)^{13}\end{array}$ & $\begin{array}{l}\text { Perfil de mortalidade por } \\
\text { quedas em idosos no período } \\
\text { de } 2000 \text { e 2010. Estudo } \\
\text { epidemiológico descritivo } \\
\text { elaborado de acordo com } \\
\text { dados do sistema de } \\
\text { informação sobre } \\
\text { mortalidade de idosos. }\end{array}$ & $\begin{array}{l}30,02 \% \text { dos óbitos foram } \\
\text { motivados por queda de } \\
\text { mesmo nível e } 24,30 \% \text { por } \\
\text { outras quedas no mesmo } \\
\text { nível. Prevaleceram a faixa } \\
\text { etária de } 80 \text { anos ou mais, } \\
\text { homogeneidade aos sexos } \\
\text { masculino e feminino, cor } \\
\text { parda, indivíduos não } \\
\text { alfabetizados e casados. }\end{array}$ & $\begin{array}{ll}\text { Estudo } & \\
\text { epidemiológico } & \\
\text { descritivo, } & \\
\text { retrospectivo, com } \\
\text { dados do Sistema de } \\
\text { Informaçãor sobre } \\
\begin{array}{ll}\text { Mortalidade entre } \\
2000 \text { a 2010. }\end{array}\end{array}$ \\
\hline $\begin{array}{l}7 \text { Jorge } \\
\text { MHPM et } \\
\text { al }(2012)^{14}\end{array}$ & $\begin{array}{l}\text { Melhorar a qualidade da } \\
\text { informação sobre a causa } \\
\text { básica de óbito devida a } \\
\text { causas externas, entre idosos } \\
\text { (60 anos e mais) no estado do } \\
\text { Rio de Janeiro, Brasil, em } \\
2006 \text {. Estudo exploratório } \\
\text { descritivo, baseado no } \\
\text { relacionamento probabilístico } \\
\text { de registros entre os bancos } \\
\text { de dados do Sistema de } \\
\text { Informações sobre } \\
\text { Mortalidade e do Sistema de } \\
\text { Informações } \\
\text { Hospitalares/Sistema Único } \\
\text { de Saúde, realizado pelo } \\
\text { software Reclink. }\end{array}$ & $\begin{array}{l}\text { Comparando-se a } \\
\text { distribuição dos óbitos por } \\
\text { tipo de causa, observou-se } \\
\text { que os óbitos por quedas } \\
(27,4 \% \text {; antes, 11,7\%) } \\
\text { praticamente triplicaram. }\end{array}$ & $\begin{array}{l}\text { Estudo exploratório } \\
\text { descritivo com base } \\
\text { no relacionamento } \\
\text { probabilístico dos } \\
\text { registros dos óbitos } \\
\text { do Sistema de } \\
\text { Informação sobre } \\
\text { Mortalidade em 2006. }\end{array}$ \\
\hline \begin{tabular}{ll}
\multicolumn{3}{l}{8 Antes DL } \\
et & al \\
$(2015)^{15}$
\end{tabular} & $\begin{array}{l}\text { Verificar a tendência de } \\
\text { mortalidade por quedas no } \\
\text { município de Florianópolis, } \\
\text { no Estado de Santa Catarina e } \\
\text { no Brasil }\end{array}$ & $\begin{array}{l}\text { No período mais recente } \\
(2005 / 2008 ; \quad 2002 / 2008 ; \\
2003 / 2008), \quad \text { houve } \\
\text { aumento significativo das } \\
\text { taxas de mortalidade } \\
\text { específica por quedas nas } \\
\text { três regiões investigadas, } \\
\text { respectivamente, e que as } \\
\text { taxas aumentaram com o } \\
\text { avançar da idade. }\end{array}$ & $\begin{array}{lr}\text { Estudo } & \text { ecológico, } \\
\text { com dados } & \text { do } \\
\text { Sistema } & \text { de } \\
\text { Informação } & \text { sobre } \\
\text { Mortalidade } & \text { entre } \\
1997 \text { a 2010. } & \end{array}$ \\
\hline
\end{tabular}

No que diz respeito ao desenho metodológico dos artigos selecionados, dentificou-se que de acordo com a metodologia de estudo, seis artigos utilizaram studos retrospectivos de série temporal com períodos entre 1996 a 2011 e tendo Sistema de Mortalidade do Ministério da Saúde como base de dados; um deles ilizou um estudo de caso/controle fatiado de outros estudos; e outro um 
exploratório pontual com relacionamento probabilístico de bases diversas. Observa-se que diversos desenhos de estudo são passíveis de avaliação do tema, o que enriquece a pesquisa temporal.

No que se refere à mortalidade geral, Rosa et $\mathrm{al}^{8}$, encontrou que a mortalidade por quedas em idosos correspondeu a 0,69\% dentre todas as causas de mortalidade no período pesquisado (2006-2011) no estado do RS. Araújo et $\mathrm{al}^{13}$, traz uma proporção de 7,15\% englobando as causas externas e Antes et al ${ }^{15}$ encontrou um percentual de $0,61 \%$. Os valores de mortalidade geral por queda em idosos mostrou-se similar nos artigos pesquisados e em regiões brasileiras distintas, o que aponta para uma mortalidade similar nos estados, sem distinção significativa para regiões brasileiras.

E em se tratando de Mortalidade Específica, Maciel et $\mathrm{al}^{9}$ encontrou que dentre os óbitos por causas externas em idosos, em média, 22,5\% se referem às quedas. Gomes et $a^{12}$ encontrou uma média de 35\%, Araújo et al ${ }^{13}$ de $29,17 \%$ e Mello-Jorge et al $^{14}$ de $14,6 \%$. Neste item, os valores encontram-se menos similares, sendo observadas variação percentual de 15 a 29\% da mortalidade específica, o que pode apontar para um subregistro da informação, uma vez que a Organização Mundial de saúde estima que as quedas respondam por aproximadamente $40 \%$ de todas as mortes relacionadas a ferimentos em idosos no mundo 2,6 .

Quanto ao Coeficiente de Mortalidade Específico por quedas (CME), Rosa et $\mathrm{al}^{8}$ encontrou um CME no período estudado de 25,94/100.000hab. Maciel et al ${ }^{9}$ encontrou um CME de 27,4 para o período. Gomes et al ${ }^{12}$ encontrou variações entre 24,06 e 35,83/100.000hab; Antes et $\mathrm{al}^{15}$ encontrou a taxa de 33/100.000hab para o ano de 2010 e os demais estudos não trazem explícito o CME nos seus rabalhos. Embora nem todos os trabalhos tragam este coeficiente, a exemplo da mortalidade específica, as taxas variam entre os estudos. 
Quanto ao gênero, Rosa et $\mathrm{al}^{8}$ aponta um maior risco para o óbito no sexo feminino; Já Maciel et $\mathrm{al}^{9}$ e Gomes et $\mathrm{al}^{12}$ concluíram que que os idosos que mais morrem por quedas são do sexo masculino; Evans et al ${ }^{10}$, Araújo et al ${ }^{13}$ e Antes et $a 1^{15}$ concluíram que não há diferenças significativas entre a mortalidade masculina e feminina, e Coutinho et al ${ }^{11}$ e Mello-Jorge et al ${ }^{14}$ não realizaram esta avaliação explícita.

Todos os estudos apontaram que quanto maior a idade, maior o risco do idoso vir a óbito quando da ocorrência queda, independente de outros fatores.

A raça branca foi apontada com maior risco de queda por Rosa et $\mathrm{al}^{8}$ e a cor parda por Araújo et $a^{13}$. Os demais artigos não realizaram esta avaliação epidemiológica.

Quanto à escolaridade, Rosa et $\mathrm{al}^{8}$ não encontrou diferença significativa nos riscos de óbitos por queda em função da escolaridade e Araújo et al ${ }^{13}$ encontrou maiores riscos na população sem escolaridade. Os demais estudos não fazem menção a esta característica.

Araújo et $\mathrm{al}^{13}$ aponta que o estado civil casado possui maior risco de óbito por queda, enquanto Rosa et $\mathrm{al}^{8}$ conclui que os idosos viúvos possuem maior risco de vir a óbito por quedas.

Todos os estudos que tiveram como base de informação os dados do Sistema de Informação sobre Mortalidade (SIM) do Ministério da saúde, apontaram para a limitação da qualidade do dado coletado, onde alguns óbitos podem estar mascarados dentro de outras causas básicas de óbito que não a queda, ou ainda informados como causas desconhecidas ou causas mal definidas de mortalidade. Esta possibilidade pode mascarar a informação real sobre a magnitude dos óbitos por quedas em idosos, prejudicando a avaliação do agravo alocação de políticas públicas que possam reduzir esta mortalidade. 
Coutinho et al11 indicam ainda que após casos de fraturas por quedas em idosos encaminhados ao hospital para tratamento, outras causas de óbito são associadas à mortalidade, tais como: pneumonia, doenças cardíacas, hemorragias, sepse, entre outras. Estas causas, informadas na Declaração de Óbito como causas básicas, podem estar mascarando o real número de óbitos que têm a queda como causa básica da mortalidade.

Esta suspeita foi demonstrada no estudo de Mello-Jorge et $\mathrm{al}^{14}$, que realizou uma comparação entre os bancos de dados de mortalidade e de internações para o município do Rio de Janeiro em 2005 e conseguiu corrigir o percentual de mortalidade específico por quedas em idosos de 11,4 para 27,4.

As evidências levantadas neste estudo destacam as quedas em idosos como um relevante problema de saúde pública na atualidade, e embora os coeficientes de mortalidade apresentem variação entre os estudos, estes se mantém num patamar alto, uma vez que o óbito por quedas é considerado como uma das causas de óbito evitáveis por atenção ao idoso, segundo lista brasileira de evitabilidade do óbito descrita por Malta et al ${ }^{16}$.

Embora instituições como a Organização Mundial de Saúde e o Ministério da Saúde estejam trabalhando com políticas de enfrentamento à ocorrência de quedas na população idosa, os resultados deste estudo indicam que o número de óbitos por quedas vem aumentando no decorrer do tempo e com o avanço da idade, o que aponta para a necessidade de melhorias na política de saúde do idoso e no enfrentamento a este problema de saúde pública 3,2 .

Este estudo possui como limitação o número restrito de artigos envolvendo a temática estudada, que pode ser explicado pelo filtro de resultado realizado neste trabalho, que pretendeu avaliar os artigos em que o óbito epresentasse o desfecho da queda, o que retirou da avaliação artigos que traziam ueda e sobrevida do idoso. 


\section{Conclusões}

Foram encontradas informações insuficientes para traçar um perfil epidemiológico da população idosa que vem a óbito por quedas, uma vez que os estudos não apontaram similaridades entre as variáveis observadas de sexo, raça/cor, escolaridade e situação conjugal, assim como disparidades entre as taxas gerais e específicas de mortalidade pelo agravo observado. Este resultado aponta para a necessidade de um olhar mais abrangente sobre este agravo em específico, visando avaliar a magnitude e o perfil destes óbitos no Brasil.

Assim, torna-se fundamental o reconhecimento que os idosos são um grupo extremamente vulnerável ao evento queda e na atuação preventiva de sua ocorrência pela equipe multiprofissional dos serviços de saúde, assim como no seu próprio domicílio, no intuito de se prevenir as quedas. A prevenção de quedas deve ser cada vez mais difundida nas discussões das políticas públicas para a saúde da pessoa idosa em especial à educação da população quanto à evitabilidade deste agravo.

Mostra-se ainda com salutar importância a qualificação da causa básica do óbito no preenchimento das informações do SIM, uma vez que a não informação da real causa básica pode mascarar a informação fidedigna sobre a magnitude dos óbitos por quedas em idosos, suas proporções e coeficientes, prejudicando a avaliação do agravo e a alocação de políticas públicas que possam reduzir esta mortalidade.

\section{Referências}

Maia BC, Viana PS, Arantes PMM, Alencar MA. Consequências das quedas em idosos vivendo na comunidade. Revista Brasileira de Geriatria e erontologia.

2011;14:381-93.

http:/ / dx.doi.org/10.1590/S1809-

98232011000200017 
2. Organização Mundial de Saúde. Relatório mundial de envelhecimento e saúde. United States of America: Organização Mundial de Saúde; 2015. Available from:

http://apps.who.int/iris/bitstream/10665/186468/6/WHO_FWC_ALC_15.01 _por.pdf?ua $=1$

3. Lei $\mathrm{N}^{\circ} 10.741, \mathrm{DE} 1^{\circ} \mathrm{DE}$ OUTUBRO DE 2003: Dispõe sobre o Estatuto do Idoso e dá outras providências, (2003)

4. Arbache J. Demographic Transformation and International Competitiveness of the Brazilian Economy. Social Science Electronic Publishing. Brasília: SSRN; 2011.^http://dx.doi.org/10.2139/ssrn.1920282

5. Nascimento JS, Tavares DMS. Prevalência e fatores associados a quedas em idosos. Texto \& Contexto - Enfermagem. 2016;25.http://dx.doi.org/10.1590/0104-07072016000360015

6. Organização Mundial de Saúde. WHO Global Report on Falls Prevention in Older Age. Unided States of America: WHO; 2007.

7. Terra NL. Entendendo as síndromes geriátricas. Rio Grande do Sul: EDIPUCRS; 2013.

8. Rosa TSM, Moraes AB, Peripolli A, Santos Filha VAV. Perfil epidemiológico de idosos que foram a óbito por queda no Rio Grande do Sul. Revista Brasileira de Geriatria e Gerontologia. 2015;18:5969.http://dx.doi.org/10.1590/1809-9823.2015.14017

9. Maciel S, Maciel WV, Teotônio PM, Barbosa GG, Lima VdGC. Perfil epidemiológico das quedas em idosos residentes em capitais brasileiras utilizando o Sistema de Informações sobre Mortalidade. Rev AMRIGS. 2010;54(1):25-31.

10. Evans D, Pester J, Vera L, Jeanmonod D, Jeanmonod R. Elderly fall patients triaged to the trauma bay: age, injury patterns, and mortality risk. The American Journal of Emergency Medicine. 2015;33(11):1635-8.

11. Coutinho ESF, Bloch KV, Coeli CM. One-year mortality among elderly people after hospitalization due to fall-related fractures: comparison with a control group of matched elderly. Cadernos de Saúde Pública. 2012;28:801-5.

Gomes LMX, Barbosa TLdA, Caldeira AP. Mortalidade por causas ernas em idosos em Minas Gerais, Brasil. Escola Anna Nery. 2010;14:779-86. 
13. Araújo AM, Menezes RMP, Mendonça AEOd, Lopes MdS, Tavares AdM, Lima HCFd. Perfil da mortalidade por quedas em idosos. Rev pesqui cuid fundam (Online). 2014; 6(3)::863-75 http://dx.doi.org/10.9789/21755361.2014v6n3p863

14. Mello-Jorge MHP, Cascão ÂM, Reis AC, Laurenti R. Em busca de melhores informações sobre a causa básica do óbito por meio de linkage: um recorte sobre as causas externas em idosos - Estado do Rio de Janeiro, Brasil, 2006. Epidemiologia e Serviços de Saúde. 2012;21:407-18.

15. Antes DL, Schneider IJC, d'Orsi E. Mortality caused by accidental falls among the elderly: a time series analysis. Revista Brasileira de Geriatria e Gerontologia. 2015;18:769-78.http://dx.doi.org/10.1590/1809-9823.2015.14202

16. Malta DC, Duarte EC, Almeida MFd, Dias MAdS, Morais Neto OLd, Moura Ld, et al. Lista de causas de mortes evitáveis por intervenções do Sistema Único de Saúde do Brasil. Epidemiologia e Serviços de Saúde. 2007;16:233-44. http://dx.doi.org/10.5123/S1679-49742007000400002 